\title{
Quantum Fluctuations and the Closing of the Coulomb Gap in a Correlated Insulator
}

\author{
A. S. Roy, ${ }^{1}$ A. F. Th. Hoekstra, ${ }^{1,2}$ T. F. Rosenbaum, ${ }^{1}$ and R. Griessen ${ }^{3}$ \\ ${ }^{1}$ The James Franck Institute and Department of Physics, The University of Chicago, Chicago, Illinois 60637 \\ ${ }^{2}$ Kamerlingh Onnes Laboratory, Leiden University, 2300 RA Leiden, The Netherlands \\ ${ }^{3}$ Division of Physics and Astronomy, Faculty of Sciences, Vrije Universiteit, Amsterdam, The Netherlands
}

(Received 11 April 2002; published 20 December 2002)

\begin{abstract}
The "switchable mirror" yttrium hydride is one of the few strongly correlated systems with a continuous Mott-Hubbard metal-insulator transition. We systematically map out the low temperature electrical transport from deep in the insulator to the quantum critical point using persistent photoconductivity as a drive parameter. Both activated hopping over a Coulomb gap and power-law quantum fluctuations must be included to describe the data. Collapse of the data onto a universal curve within a dynamical scaling framework (with corrections) requires $z \nu=6.0 \pm 0.5$, where $\nu$ and $z$ are the static and dynamical critical exponents, respectively.
\end{abstract}

DOI: 10.1103/PhysRevLett.89.276402

PACS numbers: 71.30.+h, 71.27.+a, 72.20.-i, 73.50.-h

The metal-insulator (MI) transition is only defined properly at temperature $T=0$, where the electrical conductivity $\sigma$ is identically zero in the insulator and assumes a finite value in the metal. Nonetheless, experiments at finite temperature can reveal fundamental properties of the transition by probing quantum fluctuations in the immediate vicinity of the $T=0$ critical point [1]. Quantum fluctuations inextricably link the static and dynamical response [2], and have led to predictions for new exponents and scaling forms [3] for $\sigma(T, \delta n)$ on both sides of the MI transition. Here $\delta n$ is the distance from the quantum critical point for a transition tuned by electron density, pressure, magnetic field, or any such nonthermal means.

The $T=0 \mathrm{MI}$ transition in the limit of strong electronelectron correlations has remained resistant to both theoretical understanding and experimental characterization. Progress in this direction would be welcome for illuminating the Mott-Hubbard MI transition itself as well as for shedding light on the physics of cuprate superconductors, colossal magnetoresistance perovskites, rare earth actinides, and transition metal oxides. Moreover, systematic studies of the development of correlations and fluctuations in the insulator are sorely lacking when compared to investigations of the metal. We address these shortcomings through a high-resolution study of the insulating state in yttrium hydride as we approach the $T=0$ MI transition through successive illuminations under ultraviolet light. Unlike most highly correlated materials, $\mathrm{YH}_{x}$ does not have a first-order structural phase transition that cuts off the critical behavior. It retains a continuous Mott-Hubbard MI transition, permitting full access to the quantum critical point.

Yttrium hydride and lanthanum hydride are the original "switchable mirrors" [4] with remarkable electronic and optical properties [5]. The reversible transition from shiny, metallic dihydride to transparent, insulating trihydride can be triggered at room temperature simply by changing the surrounding hydrogen gas pressure or an electrolytic cell potential [6]. A wide range of possible applications follows, from solid state displays to smart windows. A key aspect of the technology is the opening of an optical gap of order $3 \mathrm{eV}$ in the insulator [7] - a Hubbard gap - which arises from pronounced electronelectron interactions [8-10].

Conventional in situ doping by changing the hydrogen concentration is not possible at low temperatures because hydrogen diffusion, an activated process, becomes interminably slow. Instead, we tune the $T=0 \mathrm{MI}$ transition by exploiting a remarkably resilient photoconductive mechanism: charge carriers created by UV irradiation of $\mathrm{YH}_{x}$ films at $T<1 \mathrm{~K}$ persist for at least weeks to temperatures as high as $200 \mathrm{~K}$ [11]. We loaded a $550 \mathrm{~nm}$ thick Y film at room temperature to a starting hydrogen pressure $p\left(\mathrm{H}_{2}\right)=160$ bars, deep in the insulating hep $\gamma$ phase of $\mathrm{YH}_{x}$, with $x \sim 2.95$. The polycrystalline film was grown on a sapphire substrate and it is covered by $5 \mathrm{~nm}$ of Pd to enable hydrogen diffusion. The Pd cap segments into $10 \mathrm{~nm}$ wide disconnected islands that are separated from the $\mathrm{YH}_{x}$ by approximately $5 \mathrm{~nm}$ of insulating (but optically transparent) $\mathrm{Y}_{2} \mathrm{O}_{3}$, and thus cannot short circuit the intrinsic sample response. Comparison of $\sigma(T)$ between epitaxial films and bulk crystals of $\mathrm{Y}$ and $\mathrm{YH}_{2}$ indicates that the effect of uniaxial stresses from thermal mismatch with the substrate is negligible. The sample was mounted in a specially designed copper cell [12] with optical, electrical, and gas access that fits into the bore of a 14/16 T superconducting magnet of a charcoal-pumped ${ }^{3} \mathrm{He}$ system. Light from an ultraviolet stroboscope (spectral range $\sim 220-700 \mathrm{~nm}$, maximum repetition rate $10 \mathrm{~Hz}$ ) was guided to the cell by way of a UV silica fiber that illuminates the entire $7 \mathrm{~mm}$ diameter sample. The overdetermined set of van der Pauw configurations yielded consistent values of the longitudinal and Hall conductivities, indicating the good homogeneity of the sample in the $a b$ plane. Homogeneity along the $c$ axis was ensured 
by waiting for at least 1 day after hydrogenation [13], where we typically found $d \ln \sigma / d \ln t<0.01$, where $t$ is time. The measurements were carried out at frequencies between 2 and $5 \mathrm{~Hz}$ using a standard lock-in technique in the Ohmic regime.

We plot in Fig. 1 the raw $\sigma(T)$ data for a series of illuminations over three decades of conductivity and two decades of temperature. The lowest, most insulating curve with strong downward curvature is the $p\left(\mathrm{H}_{2}\right)=$ 160 bars starting point, followed by 12 successive illuminations for periods growing from $\tau=615$ to $133000 \mathrm{sec}$. The generation of charge carriers follows a $\tau^{1 / 2}$ illumination time dependence [14], which can be reset by warming to room temperature. With warming and rehydrogenation, followed by UV exposure at $T=$ $0.35 \mathrm{~K}$, it is possible to extend the dynamic range of the experiment, as represented by the upper six curves in Fig. 1. We have checked that the interleaved response from different hydrogenation and illumination cycles provides consistent data sets. In all cases, the drive parameter that we use is the charge carrier density $n$, defined in these experiments by the Hall coefficient at $T=15 \mathrm{~K}$.

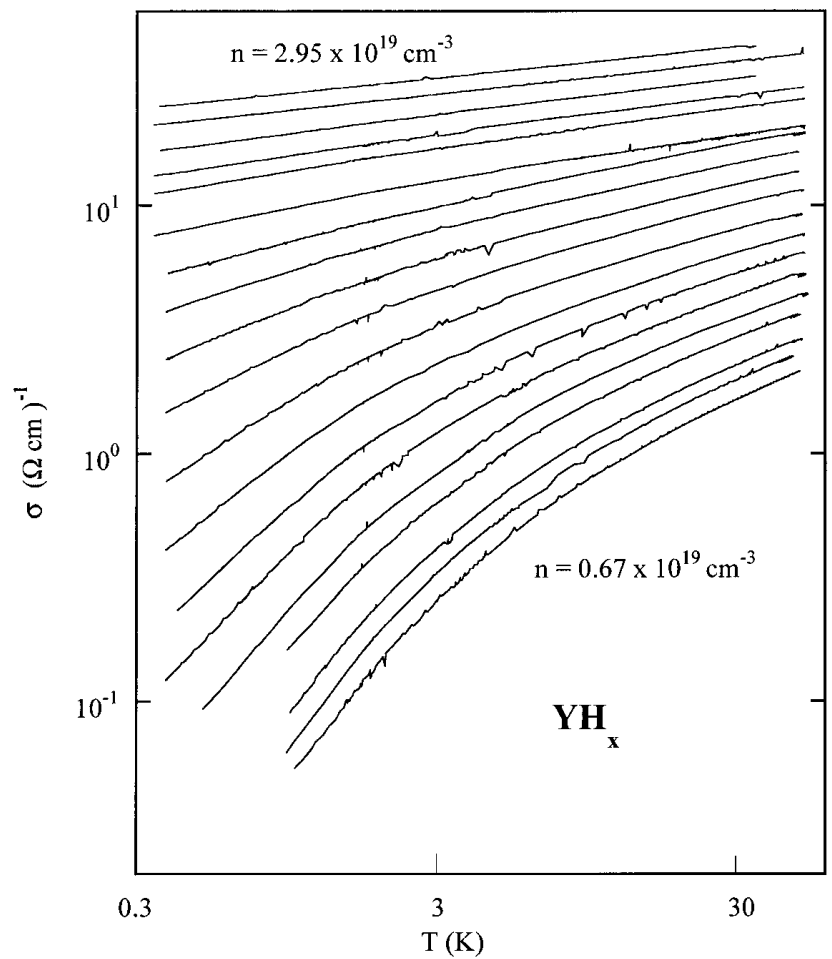

FIG. 1. Conductivity $\sigma$ as a function of temperature $T$ $(0.35<T<50 \mathrm{~K})$ for a $\mathrm{YH}_{x}$ film at a series of charge carrier densities $n(T=15 \mathrm{~K}) . n$ is systematically increased from insulator to metal through a combination of hydrogen gas loading at room temperature and UV illumination at low temperature. Unlabeled curves have $n=(0.73,0.77,0.87,0.94,1.04,1.14$, $1.24,1.36,1.53,1.65,1.82,1.97,2.04,2.32,2.43,2.59,2.83) \times$ $10^{19} \mathrm{~cm}^{-3}$.
The insulating state is distinguished by a gap in the electronic density of states that leads to activated behavior of the low temperature conductivity, $\sigma(T) \sim$ $\exp \left[-\left(T_{0} / T\right)^{\beta}\right]$. The conductivity always extrapolates to zero at $T=0$, but the finite temperature response given by the exponent $\beta$ signifies the nature of the underlying physics: $\beta=1$ for a hard gap in the density of states; $\beta=$ $1 / 2$ for a soft Coulomb gap carved out by electronelectron interactions in the presence of disorder; and $\beta=1 / 4$ for Mott variable range hopping $(\beta=1 / 3$ in two dimensions). We find that the data of Fig. 1 cannot be described by a simple activated form for any value of $\beta$ (i.e., log-log plots of $\sigma$ versus $T^{-\beta}$ display marked curvature). It is necessary to include in addition the critical (power-law) behavior, even for the most insulating of the curves:

$$
\sigma=A T^{\mu / z \nu} \exp \left[-\left(T_{0} / T\right)^{\beta}\right],
$$

where $A$ is a constant, $z$ and $\nu$ are the dynamical and static critical exponents, respectively, and the conductivity exponent $\mu$ characterizes the critical behavior of $\sigma(T=0$, $\delta n)$ in the metal. This functional form has been invoked previously to describe both insulating $\mathrm{Si}: \mathrm{B}$ [15] and $\alpha$-NbSi alloys [16].

We show in Fig. 2 the satisfactory nature of Eq. (1) for all $n$. Using the previously determined [11] critical exponents for $\mathrm{YH}_{x}, \mu=1.0 \pm 0.1$ and $z \nu=6.0 \pm 0.5$, we plot $\ln \left(\sigma / T^{1 / 6}\right)$ as a function of $T^{-\beta}$ and discover that the

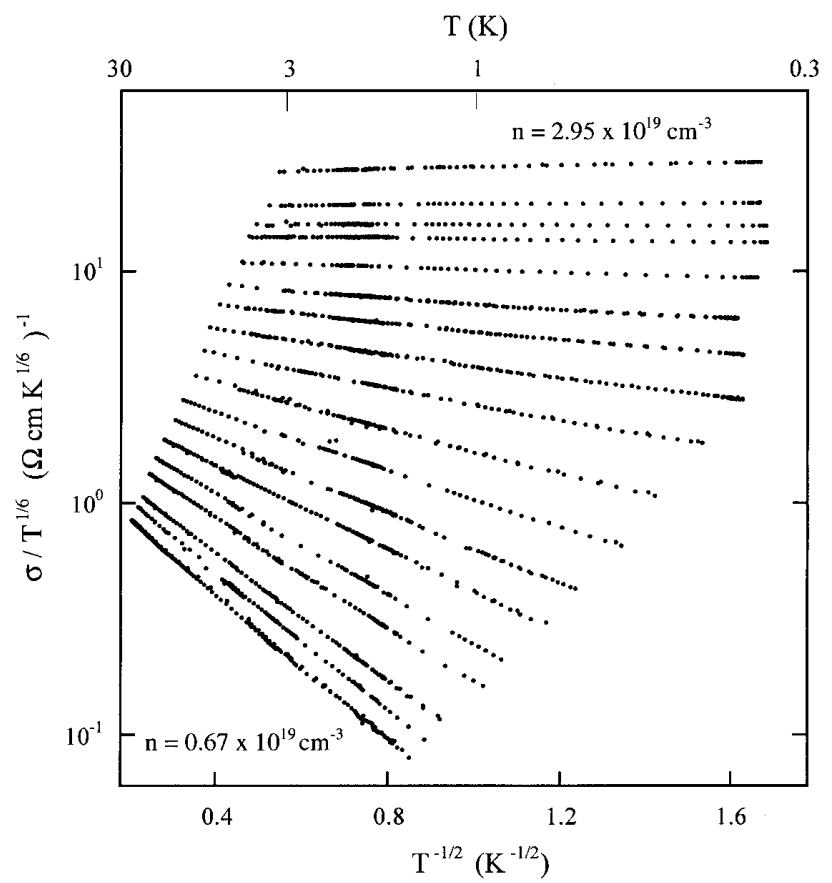

FIG. 2. The normalized conductivity $\sigma / \sigma_{c}$, where $\sigma_{c} \sim$ $T^{\mu / z \nu} \sim T^{1 / 6}$, as a function of $T^{-1 / 2}$ for the series of curves in Fig. 1. The data reflect the influence of both (power-law) quantum critical fluctuations and activated transport across the Coulomb gap $\left[\exp -\left(T_{0} / T\right)^{1 / 2}\right]$. 
data are only linear when $\beta=1 / 2$. Our conclusions are twofold: (i) Electron-electron interactions dominate the insulator in the form of a Coulomb gap and Efros-Shklovskii hopping transport [17], and (ii) the measurable effects of quantum fluctuations - an unconventional $T^{1 / 6}$ spectrum-extend surprisingly far from the critical point. A crossover to pure hopping behavior presumably occurs at lower temperatures than those probed in this experiment.

The Coulomb gap scale, $T_{0}$, is related to the dielectric constant $\kappa$ and the localization length $\xi$ by the relation $k_{B} T_{0}=\left(1 / 4 \pi \varepsilon_{0}\right)\left(2.8 e^{2} / \kappa \xi\right)$, where $\kappa$ and $\xi$ are implicit functions of $n$. Both $\kappa$ and $\xi$ diverge at the approach to the MI transition from below, and

$$
T_{0}\left(n<n_{c}\right) \propto\left(1-n / n_{c}\right)^{z \nu}=\left(\delta n / n_{c}\right)^{z \nu} .
$$

$T_{0}(n)$ can be read from the slopes of the curves in Fig. 2 and we plot its sharp drop with increasing charge carrier density in Fig. 3. The solid line is a fit to Eq. (2), where we fix $z \nu=6.0$ and solve for best fit value $n_{c}=2.8 \times$ $10^{19} \mathrm{~cm}^{-3}$ [18]. The measurements do not extend to sufficiently low temperatures to determine the characteristic Coulomb gap temperatures for $n \sim n_{c}$. Hence, the critical behavior of $T_{0}\left(\delta n / n_{c}\right)$ serves as a self-consistency check rather than as an independent determination of the critical exponents.

A more stringent test of the dynamical scaling framework involves the ability to collapse all the data on a universal curve. Previous attempts to scale $\mathrm{YH}_{x}$ data very close to $n_{c}$ have succeeded in the metal, but failed in the

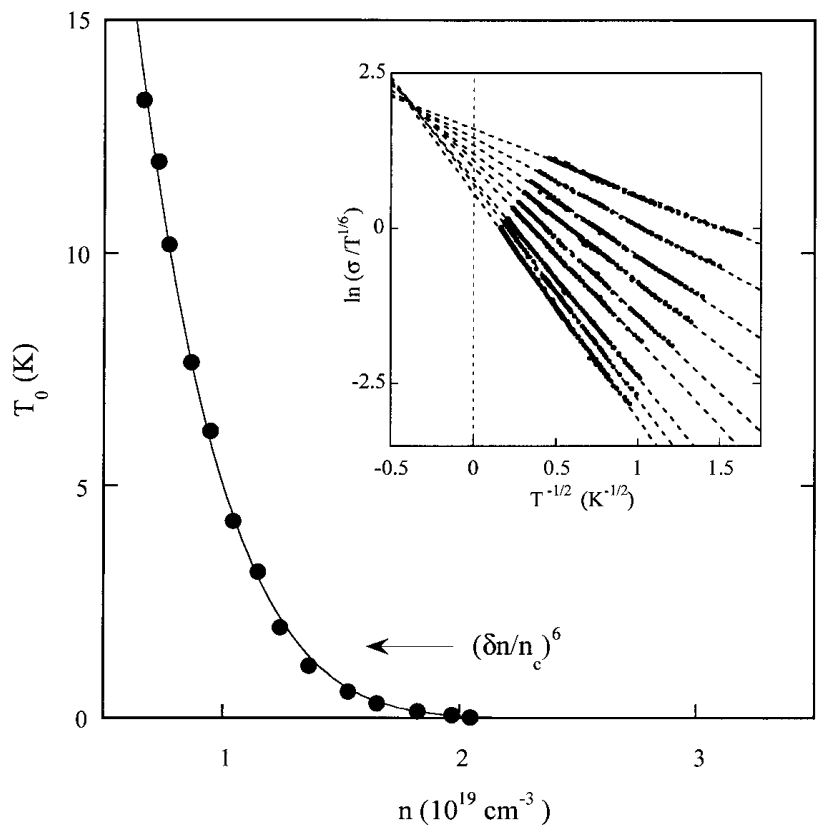

FIG. 3. The Coulomb gap scale $T_{0}$ vanishes as $\left(\delta n / n_{c}\right)^{z \nu}$, with $z \nu=6$. Inset: Straight line fits to the nine most insulating curves of Fig. 2 do not intersect as expected at the abscissa zero, but at a common point $(-\alpha, \phi)$ with $\alpha=0.39$. insulator [11]. Using the extended data set of this experiment, we are able to discover why. We focus in the inset to Fig. 3 on the nine most insulating curves with $0.55 \mathrm{~K} \leq$ $T_{0} \leq 13 \mathrm{~K}$. In all cases $T_{0}$ exceeds the base temperature of the cryostat $(0.35 \mathrm{~K})$ and Eq. (1) is strictly valid. The points should fall on straight lines with slopes $-T_{0}^{1 / 2}$ and a common $y$ intercept equal to $\ln (A)$. It is clear from the figure that $A$ is not a constant, but varies systematically with $n$. However, the curves do intersect at one point when extrapolated (dashed lines) to a negative value of $T^{-1 / 2}$.

The usual scaling function for $\sigma(T, \delta n)$ is of the form $T^{1 / z \nu} \mathcal{F}\left(\delta n / T^{1 / z \nu}, \lambda T^{\gamma}\right)$, where both $T$ and $\delta n \rightarrow 0$ at the quantum critical point. The intersection of the data in Fig. 3 at a negative intercept $-\alpha$ requires a scaling function that is the product of two terms, $\exp \left[-\alpha T_{0}^{1 / 2}\right] \times$ $\exp \left[-\left(T_{0} / T\right)^{1 / 2}\right]$, with $T_{0} \sim(\delta n)^{z \nu}$. The insulating conductivity is then captured by the function (with $\mu=1$ ):

$$
\sigma(T, \delta n) \sim T^{1 / z \nu} \exp \left\{-\left(\delta n^{z \nu} / T\right)^{1 / 2}\left(1+\alpha T^{1 / 2}\right)\right\},
$$

where we have made explicit the corrections to scaling. This function leads to the collapse with little scatter of all nine data sets on a universal curve (Fig. 4).

The existence of a correction term is not surprising given the distance of the data from the critical point, although its particular form remains theoretically obtuse. Quantitative analysis closer to the MI transition will require lower temperature experiments in order to continue to capture the interplay of the Coulomb gap and quantum fluctuation physics. We note, that unlike the phenomenology near the quantum Hall-to-insulator transition [19], scaling here remains critical. The ability to treat the insulator on an equal footing with the metal

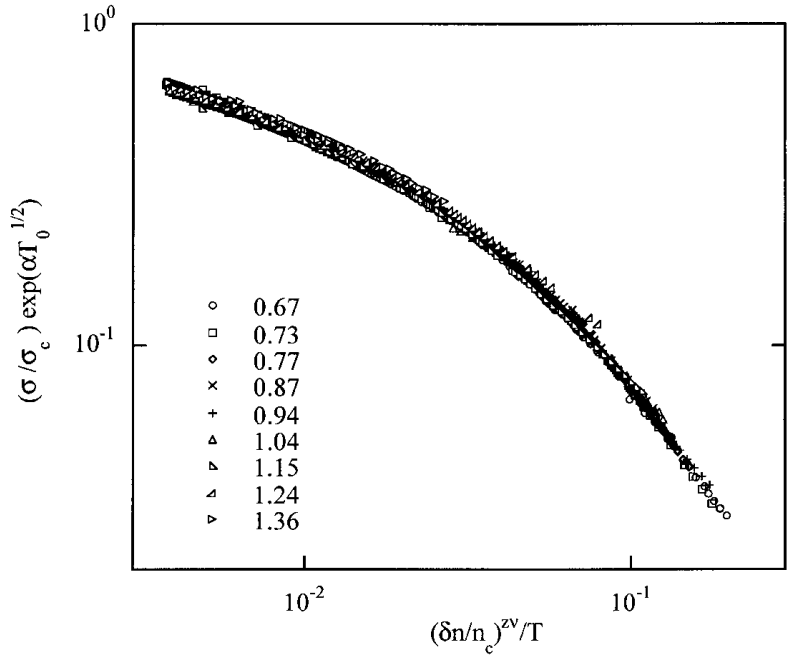

FIG. 4. Collapse of the data on a universal curve using dynamical scaling with corrections [Eq. (3) in the text]. Carrier density $n$ for each data set is indicated in the legend in units of $10^{19} \mathrm{~cm}^{-3}$. 
lends credence to the unusually large product of the static and dynamical critical exponents $(z \nu=6.0 \pm 0.5$ for $\mathrm{YH}_{x}$ and $z \nu=4.6 \pm 0.4$ for the Mott-Hubbard transition metal compound $\mathrm{NiS}_{2-x} \mathrm{Se}_{x}$ [20]) that appears to distinguish continuous MI transitions in the highly correlated limit.

We are indebted to S. M. Girvin and S. Sachdev for explaining the nuances of the scaling theory, to R. J. Wijngaarden for scientific advice, and to B. Dam, S. Enache, and N. J. Koeman for technical assistance. The work at the University of Chicago was supported by NSF DMR-0114798.

[1] S. Sachdev, Quantum Phase Transitions (Cambridge University Press, Cambridge, 1999).

[2] J. A. Hertz, Phys. Rev. B 14, 1165 (1976); A. J. Millis, Phys. Rev. B 48, 7183 (1993).

[3] S. L. Sondhi, S. M. Girvin, J. P. Carini, and D. Shahar, Rev. Mod. Phys. 69, 315 (1997).

[4] J. N. Huiberts et al., Nature (London) 380, 231 (1996).

[5] For recent reviews, see R. Griessen, Europhys. News 32, 41 (2001); T. F. Rosenbaum and A. F. Th. Hoekstra, Adv. Mater. 14, 247 (2002).

[6] F. J. A. den Broeder et al., Nature (London) 394, 656 (1998).

[7] A. T. M. van Gogh et al., Phys. Rev. B 63, 195105 (2001).
[8] R. Eder, H. F. Pen, and G. A. Sawatzky, Phys. Rev. B 56, 10115 (1997).

[9] K. K. Ng et al., Phys. Rev. Lett. 78, 1311 (1997); Phys. Rev. B 59, 5398 (1999).

[10] P. van Gelderen, P. A. Bobbert, P. J. Kelly, and G. Brocks, Phys. Rev. Lett. 85, 2989 (2000).

[11] A. F.Th. Hoekstra, A.S. Roy, T.F. Rosenbaum, R. Griessen, R. J. Wijngaarden, and N. J. Koeman, Phys. Rev. Lett. 86, 5349 (2001).

[12] A. F. Th. Hoekstra, T. F. Rosenbaum, and A. S. Roy, Rev. Sci. Instrum. 73, 119 (2002).

[13] S. J. van der Molen et al., J. Appl. Phys. 86, 6107 (1999).

[14] A. S. Roy et al. (to be published).

[15] S. Bogdanovich, M. P. Sarachik, and R. N. Bhatt, Phys. Rev. Lett. 82, 137 (1999).

[16] H.-L. Lee, J. P. Carini, D. V. Baxter, W. Henderson, and G. Grüner, Science 287, 633 (2000).

[17] A. L. Efros and B. I. Shklovskii, J. Phys. C 8, 249 (1975).

[18] The value $n_{c}(T=15 \mathrm{~K})=2.8 \times 10^{19} \mathrm{~cm}^{-3}$ cannot be compared directly to the $n_{c}(T=0.35 \mathrm{~K})=1.39 \times$ $10^{19} \mathrm{~cm}^{-3}$ reported in Ref. [11] because of the different temperatures at which the Hall data were collected. As the Hall coefficient varies smoothly through $n_{c}$ at any $T$ in this temperature range, the observed scaling behavior and critical exponents are unaffected.

[19] D. Shahar et al., Solid State Commun. 107, 19 (1998).

[20] A. Husmann et al., Science 274, 1874 (1996); Phys. Rev. Lett. 84, 2465 (2000). 